\title{
Oral Manifestations Following GERD, Self-Injury Behavior and Wearing Dental Removable Prosthetics: An Overview and a Case Report
}

\author{
Pierluigi Guerrieri $^{1 *}$, Andrea Oliveira ${ }^{2}$, Federico Arosio ${ }^{1}$, Martino Meuli $^{3}$, Luca Viganò ${ }^{2}$, Cinzia Casu $^{4}$
}

${ }^{1}$ IRCCS Ca Granda Fondation General Hospital, University of Milan, Milan, Italy

${ }^{2}$ DDS, San Paolo Dental Building, University of Milan, Milan, Italy

${ }^{3}$ DDS, Departement of Surgical, Pediatric and Diagnostic Science, University of Pavia, Italy

${ }^{4}$ DDS, Department of Surgical Science, OBL Oral Biotechnology Laboratory, University of Cagliari, Italy

DOI: $10.36347 /$ simcr.2020.v08i12.015

| Received: 13.12.2020 | Accepted: 24.12.2020 | Published: 29.12.2020

*Corresponding author: Pierluigi Guerrieri

Abstract

Introduction: Some physical and mental conditions can directly or indirectly influence the health of the oral cavity especially for traumatic injuries. In this review we decided to consider the presence of dental prostheses, mental illnesses and even gastroesophageal reflux in the development of traumatic lesions of the oral cavity. All these conditions are characterized by a greater risk of developing injuries. The aim is also to report a case of tongue erosion from gastro-esophageal reflux successfully treated with $808 \mathrm{~nm}$ diode laser. Materials and Methods: We investigated PubMed database using the keywords: "Oral manifestation of gastrointestinal disorders" "mental retardation and oral biting" and "denture wearers' oral lesion". Where possible, we included systematic review. Results: Several scientific papers, including systematic reviews, have highlighted the correlation between gastroesophageal reflux and oral lesions, such as dental erosion, erythema in various sites, glossitis and periodontitis. Mental retardation is involved in the development of self-injuries at the oral level, which may also evolve into serious lesions such as oral carcinoma. The most common denture-related oral lesions are fibrous hyperplasia, traumatic ulcers and denture stomatitis. Poor oral hygiene and inadequate fitting of the dentures appear to be risk factors for these injuries. Conclusion: It is important to evaluate the adequacy of dental prostheses in order to avoid traumatic mucosal injuries. It is also necessary to control gastric acidity to reduce the risk of developing lesions. For patients with mental disability, a close follow up and the use of intraoral devices is advisable. The use of diode laser could be a very safe and interesting tool for treatment of symptomatic oral mucosal lesions from GERD.

Keywords: Oral manifestation of GERD, oral traumatic lesions, oral lesions from prosthesis, oral lesions from mental retardation, $808 \mathrm{~nm}$ diode laser.

Copyright $\odot 2020$ The Author(s): This is an open-access article distributed under the terms of the Creative Commons Attribution 4.0 International License (CC BY-NC 4.0) which permits unrestricted use, distribution, and reproduction in any medium for non-commercial use provided the original author and source are credited.

\section{INTRODUCTION}

Many medical conditions, both organic and functional, can increase the risk of developing injuries in the oral cavity. Some of these conditions may occur clinically as oral manifestations in the early or advanced stages of the disease. This paper aims to first examine the onset and development of oral lesions due to the disease and symptoms of gastro-esophageal reflux (GERD/S) and mental disorders associated with selfinjury and compulsive behavior. Also, we assess some of the oral lesions that occur after the application of a removable prosthesis.

We considered GERD/S in the development of oral injuries mainly since its prevalence has been increasing since 1999 and is one of the most common gastrointestinal diseases in the United States and a global burden on health systems [1]. GERD is a multifactorial disease. GERD is consistently present in Italy, the country from which we are writing, and is common in both sexes [2].

It is not uncommon for oral manifestations attributable to GERD to occur in the young, adult, and elderly. GERD can be considered a relevant economic, social, and quality of life burden since the illness consequences intersect all these dimensions [3]. Knowing and estimating risk factors is very important in the prevention of gastroesophageal reflux and related oral injuries.

This article presents a clinical case of a man affected by a mucosal disorder of his tongue, which is associated with GERD. The clinical case is an example of the approach often mentioned as "dental management of the medically compromised patient". It 
is known, in fact, that gastrointestinal conditions can affect the oral and dental health of patients. The oral manifestations of the disease can potentially guide the diagnosis and support the identification of the medical condition of origin.

Besides, we decided to investigate oral lesions resulting from chronic and compulsive self-harm behavior in patients with mental and neurological disorders. Some of these patients, such as those suffering from specific genetic syndromes, often exhibit self-injurious behavior, which may compromise oral tissues integrity and, globally, oral-dental health. It needs to be considered that mentally ill patients may be purposely hospitalized owing to a dental condition [4]. The dentist may be involved in the multidisciplinary context of the therapy, for example, to design intra-oral protective devices that can prevent self-mutilation trauma. However, it is necessary to consider that these devices must be carefully designed to prevent additional damage. Protective intra-oral devices of such kind may be iatrogenic or interfere with behavioral and nonbehavioral factors of these patients.

Concerning the relationship between quality of life and oral health of the patients, we decided to investigate oral iatrogenic lesions attributable to incorrect use and maintenance of removable dentures. Total or partial edentulousness, together with removable dentures, can influence the quality of life and nutritional function of the individuals $[5,6]$. Since oral health is closely related to the quality of life, it is important to know whether removable dentures are related to the development of oral injuries. We aim to highlight that denture-related lesions may be associated with the iatrogenic effect of the prosthetic device and the local and general factors of the denture wearers.

We investigated PubMed database using the keywords: "Oral manifestation of gastrointestinal disorders" "mental retardation and oral biting" and "denture wearers' oral lesion". Where possible, we included systematic review.

\section{GERD-ASSOCIATED ORAL DISORDERS: A SHORT REVIEW AND A CASE}

Gastroesophageal reflux disease (GERD) is a condition that develops when gastric contents flow retrogradely into the esophagus causing wide-ranging symptoms and complications. The prevalence of weekly symptoms in the US population increased by about $50 \%$ until 1995 [1]. GERD depends on an extensive combination of risk factors, both hereditary and environmental. Recognized risk factors include obesity, dose-dependent tobacco smoking, pregnancy, hiatal hernia, some classes of drugs, and the consumption of certain beverages or foods. The clinical evaluation of patients should immediately lead to identifying signs and symptoms, both typical and atypical ones [7].
Some studies report that GERD patients have a significantly higher risk of suffering from xerostomia and reduced swallowing function [8]. Together with the action of acid reflux and increased risk of susceptibility to oral infections, these findings lead to the onset of oral symptoms in patients with GERD. Oral symptoms can be distinguished into dental erosion and oral soft tissue disorders [9]. According to the Montreal Definition and GERD classification, dental erosion is considered an important oral phenomenon in these patients. It is defined as the irreversible loss of dental tissue mediated by a chemical process without the involvement of bacterial factors [10]. In GERD patients, dental erosion occurs as the predominant oral lesion outside the esophageal area and represents an atypical manifestation of the disease [11]. Patients with dental erosion may require extensive dental therapy to overcome the aesthetic and functional impairments [12]. Bartlett et al., evaluated the relationship between GERD, oral $\mathrm{pH}$ value change, and dental erosion. The authors measured the oral $\mathrm{pH}$ and the esophageal $\mathrm{pH}$ simultaneously and found that a $\mathrm{pH}$ measurement below 5 at both sites was related to palatal erosion. Gastric acids, in fact, flow from the stomach to the esophagus and then to the oropharynx [13]. A systematic review of 2018 by Picos et al. analyzed the association between GERD and dental erosion in adults and children. They noted that the prevalence of dental erosion in patients with GERD was $48.81 \%$, while it was $20.48 \%$ in nonGERD controls. Furthermore, the prevalence of dental erosion in children with GERD was higher than in adults within the same conditions [14]. Another systematic review by Pace et al. reached the same result highlighting the strong correlation between GERD and dental erosion [15].

Dental erosion is a multifactorial phenomenon. It is attributable to a decrease in $\mathrm{pH}$ values, but also the reduced capacity of salivary buffering systems within GERD patients [12]. The pathophysiological mechanism underlying dental erosion includes chemical and electrolytic processes. Gastric acids regurgitated towards the oral cavity have a $\mathrm{pH}$ below 2.0. This value below the threshold limit of 5.5 can dissolve the hydroxyapatite crystals of the enamel [16]. The palatal surfaces of teeth are the most affected. The lingual surfaces of teeth can also be associated with acid erosion. The explanation lies in the fact that these surfaces are located within the path of the material flowing from the pharynx into the oral cavity [17]. All factors that increase the duration of contact of gastric substances with teeth predispose to dental erosion risk [16].

Reduced salivary flow volume in patients with GERD may reduce the protection exerted by the salivary fluid on gingival soft tissues and mucosal lining. A relationship between gingivitis and GERD has been suggested. The reduction in salivary flow may act as an exacerbation factor of the gingivitis combined 
with GERD [18]. Besides, further authors have reported inflammation of oral mucosal regions in patients with GERD, including the tongue, oral floor, vestibule, and oral mucosa lining. The most reliable hypothesis to explain the lesion of oral mucosal regions when associated with GERD is that they are damaged by the direct action of the gastric acid refluxate in the oral cavity, particularly on the palate [9].

A 2018 study by Shimazu et al., carried out on rats put in evidence oral changes in an experimental rat model of GERD. Here too dental erosions, glossitis and periodontitis were observed [19].

It was reported that patients with GERD show a higher prevalence of bruxism. In turn, bruxism is a traumatic risk factor that may exacerbate gingivitis and inflammatory lesions of the oral mucosa. GERD patients and even bruxists may experience a very rapid loss of dental integrity associated with acid erosion [12].

\section{CASE REPORT}

A 62-year-old man visited our private practice. His medical history showed a pre-existing gastroduodenal ulcer, hiatal hernia, and esophagitis attributable to GERD/S. The patient suffered from slight arterial hypertension. The general state of oral hygiene was excellent. There were neither carious lesions nor periodontal pathologies. The patient showed some defects of the dental hard tissue of erosive nature. Dental erosion was presumably caused by a variation in saliva $\mathrm{pH}$ due to frequent acid regurgitation. After an acute episode of esophagitis, the patient also showed alterations of the oral soft tissues, mainly of the tongue. The clinical inspection of the lingual mucosa showed areas of reduction in thickness attributable to tissue erosion, and hypertrophic areas. The hypertrophic areas were mainly concerned with the filiform papillae (Fig1). No ulcerative lesions were found either on the tongue or on the palate, which is one of the areas where oral GERD lesions usually occur.

Considering our clinical experience, which often uses a diode laser for oral soft tissue procedures, we immediately provided the patient with this option. Before the procedure was started, thus, the lingual body was again clinically evaluated and slightly tensioned, as suggested by Pirnat, to both identify the hypertrophic regions to be treated and to evaluate the direction of application of the laser. A diode laser of $808 \mathrm{~nm}$ of wavelength, with a power of 250 mwatt and a spot of 5 $\mathrm{cm}^{2}$ was used. The use of diode laser resulted in the photobiomodulation and disinfection of the treated lingual regions. At 1 week follow up the patient was free of symptoms and signs (Fig-2). The distance of the spot from the tongue was about $1.5 \mathrm{~cm}$. In this way, the beam could pass through the entire epithelial thickness with no iatrogenic thermal damage [20].

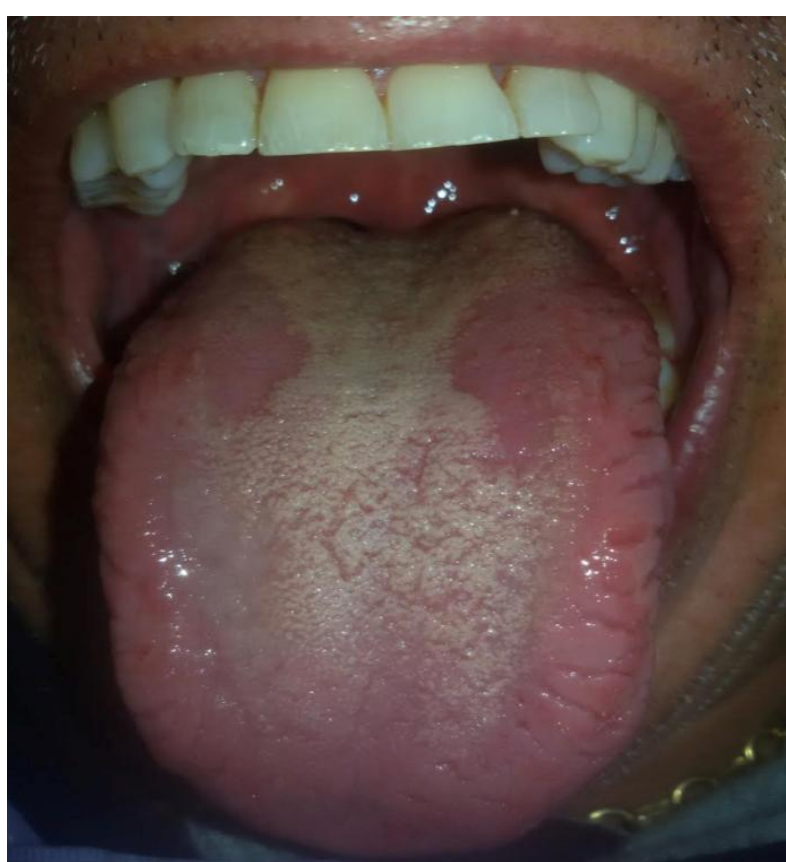

Fig-1: The anterior-upper surface of the tongue of the patient showed two bilateral erosive areas. Among these areas a hypertrophy of the lining epithelium and mainly of the filiform papillae was evident. At the margins, spare fissures and some signs of irritation were detected.

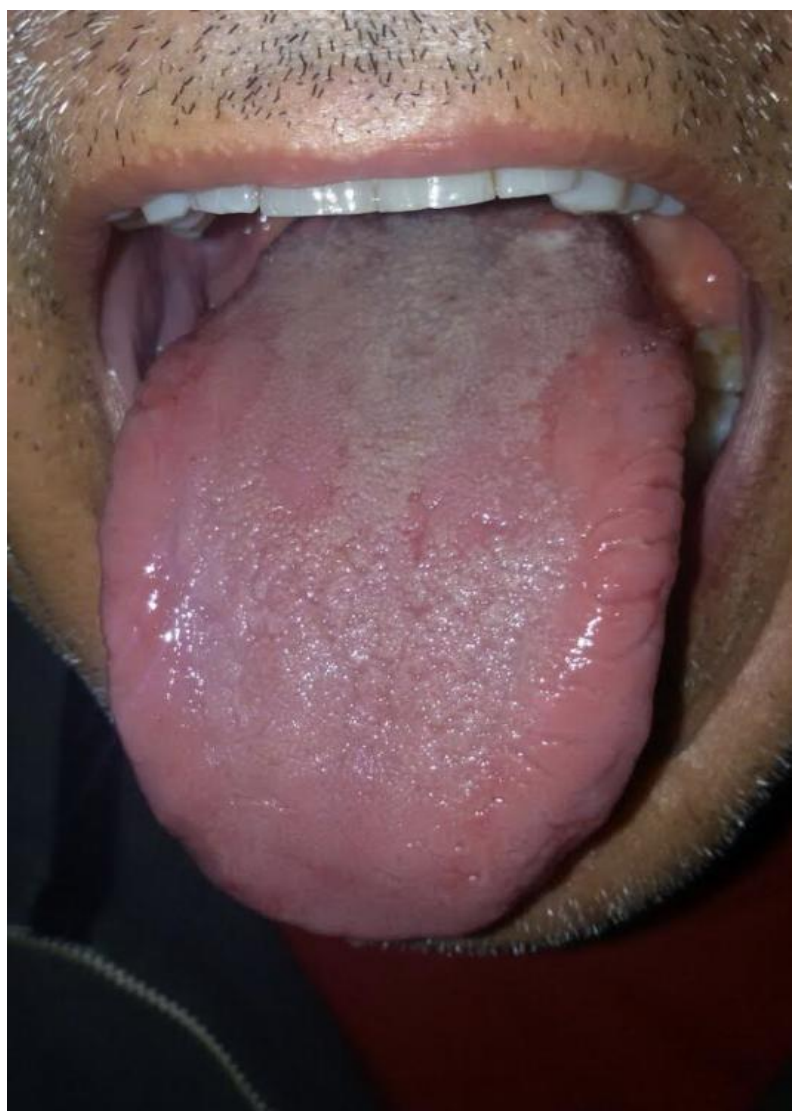

Fig-2: The anterior-superior surface of the tongue of the patient underwent a healing phase after laser treatment at 1- week clinical follow-up. The patient had neither symptoms nor tongue irritations 


\section{SELF-INJURY ORAL LESIONS: A SHORT} REVIEW

Self-mutilation or self-injury are behavioral disorders that result in the destruction or alteration of some parts of the human body without suicidal intent. Acts of self-harm are often directed towards the regions of the head, neck, shoulders, and hands [21, 22]. Traumatic and self-harm behavior can depend on organic or functional causes. Organic aetiologies include genetic syndromes, biochemical-enzymatic deficiencies, and some neurological disorders [23, 24]. In these cases, the patient experiences repeated and compulsive trauma against himself, but without conscious intention. Functional self-harm derives instead from some conscious stimuli and does not come from an organic background [23]. Therefore, it is necessary to place the clinical case into the differential diagnosis with multiple psychiatric disorders. In this review, we want to focus on the importance of oral inspection in patients suffering from various neuronalpsychiatric diseases, supposed causes of self-inflicted injuries of organic nature. Some authors have reported the main diseases associated with a higher risk of compulsive self-harm behavior. Genetic syndromes include, for instance, Lesch-Nyhan syndrome, Tourette syndrome, and Cornelia de Lange syndrome.

Concerning the elderly population, most oral self-harm behaviors are linked to degenerative disorders such as Parkinson's disease and Alzheimer's disease [23]. Other conditions include mental retardation, autism, congenital malformations, congenital infectious diseases, epilepsy, and hereditary sensory neuropathies. Injuries directed against the oral cavity can frequently affect the tongue and lips and involve dental tissue, periodontal tissue, and mucous membrane.

Recently, Okabe et al., reported the case of a 40-year-old woman suffering from Cornelia de Lange syndrome. The behavior detected was a chronic and compulsive self-biting phenomenon that had generated injury to her right buccal mucosa. The authors, immediately after the hospitalization of the woman, reported to have applied a splint protection made of ethylene-vinyl-acetate on her upper arch [25].

Among the various genetic syndromes that can lead to oral self-harm disorders of organic nature, we recall the Lesch-Nyhan syndrome. The syndrome was first described in 1964 and is a recessive X-related disorder. In these patients, uric acid production is increased, and an excess of urate crystals deposit within the peripheral organs. This effect results in various symptoms, as well as mental retardation and neurological and musculoskeletal manifestations. Of the various symptoms, self-mutilation of the lips and the tongue is frequent [22]. The lower lip may be often totally or partially destroyed as a result of chronic mosicatio labiorum. Chronic bites on the tongue and lips can cause local ulceration, nodular traumatic lesions and local infections. Cases of self-amputation of the tongue were described [22].

Regarding oral lesions in mentally ill patients, Siragusa et al. assessed the presence of oral lesions in 245 patients admitted to a psychiatric institution. Numerous lesions involving the tongue, lips, oral mucosa, and teeth were diagnosed. They were mainly due to a mechanism of self-mutilation (morsicatio buccarum, morsication labiorum, cheek biting, tongue biting) [26].

A continuous traumatic action on the soft tissues can result in the evolution of early lesions into a carcinoma. Kashyap et al. reported the case of an 85year-old patient with mental health disorders who selfinflicted repeated dental injuries. This continuous trauma to the lip resulted into the development of a carcinoma [27].

A promising treatment to prevent these injuries is represented by intraoral protective devices. It may be helpful to require a multidisciplinary approach and refer the patient to expert medical care and an orthodontist. An intraoral device can act as a protective shield against compulsive trauma and, therefore, is useful to prevent the loss of soft tissues and the integrity of the dental arches of the patients. The therapeutic action mechanism involves either increasing the vertical dimension or the physical separation of soft tissues from the adjacent dental arch sector. In some cases, restoring the vertical dimension already allows soft tissue not to penetrate the inter-occlusal space [28]. The construction of an intra-oral device requires careful occlusal and temporomandibular evaluation.

It is always necessary to place self-damaging behaviors into differential diagnosis with neuropathological mastication, which is caused by the uncoordinated myotonic activity of the chewing muscles and tongue [29].

If the patient is pediatric, the differential diagnosis may be more difficult. Also, these patients do not fully understand their physical and mental condition, and it is more difficult for them to control it individually. Psychotic conditions are the main cause of injuries [30]. Treatment with acrylic oral devices is conservative and should require the cooperation of the parents or the caregivers of the patients, especially concerning oral hygiene that may be lacking in people with motor deficits.

However, there are no standardized treatment protocols, and therefore, therapy must always be individualized. Treatment can be classified in 4 different modalities: psychological therapy, drug therapy, intraoral devices, and surgical therapy [21, 24]

About treatment, a 2008 work by Santos et al. highlighted many ways to control self-inflicted trauma 
to the oral cavity, including a neuromuscular block due to type A botulinum toxin. The authors reported that its use is safe, well-tolerated, and effective in alleviating muscle spasticity, bruxism, and lip bites [31].

\section{DENTURE-RELATED MUCOSAL LESIONS: A SHORT REVIEW}

Removable prostheses require certain features to ensure good patient satisfaction. The prosthesis must have good retention, support and stability properties [32]. Many factors can influence the retention of the prosthesis [33], and many others, related to the local or systemic conditions of the patient, contribute to the clinical success of the rehabilitation.

According to Budtz-Jorgensen et al, oral mucosal lesions, if associated with a prosthetic device, may constitute a heterogeneous group depending on their pathogenesis. If the tissue damage is mainly caused by the prosthesis, it is probably attributable to the traumatic-mechanical action of the denture on the oral tissues of the patients and to the loading effect of the base of the prosthesis on the oral mucosa [34]. Unbalanced occlusion occurs as another risk factor [35].

Denture lesions may also result from nonprosthetic factors, assuming the fact that the denture is properly fabricated. However, the denture may act as a trigger to slatentize or exacerbate a local condition.

For injuries related to dentures, a 2015 work by Mubarak et al., examined 210 patients, including 166 males. Injuries were found in $20.5 \%$ of cases. The most frequent lesion ( $41.9 \%$ of cases) was found to be fibrous hyperplasia. However, the author pointed out that the results may vary from study to study, as much depends on the type and quality of the material used for the prosthesis [35]. Fibrous hyperplasia is an exophytic lesion and can be removed surgically, by laser, mucoabrasion or cryotherapy [36]. These authors argued that the lesion originates from chronic and repeated trauma, but at low intensity. Mubarak et al., asserted that the redundant tissue comes from a prosthesis that is neither well retained nor stable. Another causative factor may be the thin edge of a flange. Other factors, both microbial and salivary, are not excluded [35].

Another type of injury attributable to the denture is traumatic ulcers, also known as decubital ulcers.

Jainkittivong et al., also wanted to study the prevalence of mucosal lesions related to dental prosthesis (DML). They observed in a sample of 380 denture wearers that $45 \%$ of denture wearers had DML and that the difference in the prevalence of oral lesions between total and partial denture wearers was not significant (49\% versus $42.2 \%$ ). They observed that the most common DMLs are traumatic ulcers (19.5\%).
They are often diagnosed both in patients wearing partial removable partial and total dentures [37].

These lesions have a multifactorial etiology and are caused by factors related to the prosthesis and the patient (local and systemic factors). Prosthetic factors include irregular surfaces, bases or extensions in the body of the device and incorrect fabrication with regard to occlusion, vertical size dimension and patient anatomy. Local and systemic factors can be summarized as follows: lack of mucosal support, irregular bone crests, exostosis, diabetes and immune dysfunction. The original damage is purely mechanical during chewing, phonation and even during sleep. The therapy consists of removing the irritant source. If the lesion does not regress even when the associated etiological agent is removed, the lesion must be placed in differential diagnosis with other ulcerative diseases of the oral cavity, and a biopsy must be performed [38].

Care must be taken not to misdiagnose a denture lesion instead of a squamous cell carcinoma of the oral cavity. Valente et al. reported the case of a 65 year-old woman suffering from a mucosal ulcer of the alveolar crest. The injury was related to the presence and wearing of a new complete mandibular prosthesis. In fact, the therapy began by advising the patient to stop using the prosthesis for 1 week and to apply topical lidocaine three times a day to relieve the pain. The lesion persisted even after the first attempt of the therapy, and then, a biopsy was indicated. Histological examination confirmed an ulcerative and poorly differentiated oral carcinoma [39].

Interestingly, our bibliographic review showed the case of a 66-year-old man from South Korea who showed an ulcerative injury located on her upper lip and probably due to the presence and wearing of a complete denture. In fact, a prosthesis had been worn for 10 years, but the lesion had remained in the anterior maxillary vestibule for about 5 months. The biopsy revealed granular inflammation caused by tuberculosis. In this case, the authors wanted to emphasize that oral tuberculosis mimicked a denture traumatic ulcer [40].

Denture stomatitis is another disorder that may potentially affect denture wearers. Although it is quite common as a disorder, its aetiology is not entirely clear. The risk factors are related to poor oral hygiene, the presence of plaque and poor adhesion of the denture on the oral mucosal tissues. The development of new materials should prevent bacterial biofilm from adhering to the denture, thus improving the level of hygiene.

Denture stomatitis is an inflammatory process of the regions of the oral mucosa that are at the base of a removable denture or under its edges. It can affect 15$70 \%$ of edentulous patients who need conventional therapy with a removable prosthesis for functional and 
aesthetic purposes. The primary aetiology seems to be microbial but the salivary, metabolic, pharmaceutical, nutritional and endocrine factors must also be considered. Denture stomatitis is also known as chronic erythematous candidiasis and is often localized at the palate level.

A persistent microbial biofilm initiates subprosthetic tissue damage on the surface of the prosthesis. This biofilm is a protective site for some microorganisms such as C. albicans. C. albicans is considered the primary etiologic agent because it can adhere to the prosthetic resin and benefit from the anaerobic environment between the prosthetic base and the palate of the patient [41]. According to Ramage, these microorganisms can easily adhere to imperfections and cracks in the prosthetic material [41, 42]. The fungal colonization is based on the porous surface of the acrylic resin. The prosthetic bases thus serve as reservoirs for these microorganisms. Cigarette smoke may predispose to colonization by altering the epithelium and providing nutrients for fungal growth and proliferation [43].

The hygiene of the prosthetic device is essential to decontaminate the prosthetic surfaces that interact with the tissues of the patients. The prosthesis should be brushed and cleaned with oral disinfectants. It is necessary to instruct the patient in the maintenance and cleaning procedures of their dentures, as well as the most appropriate use during the day. Denture stomatitis may be treated with antifungal agents and the therapy should always remove all the etiological factors involved [41, 44, 45]. The most frequently used pharmacological agents are miconazole and nystatin. Photodynamic antimicrobial therapy is a rather valid treatment alternative [46].

Other lesions associated with wearing dentures and which may depend on a multifactorial etiological combination are angular cheilitis, friction keratosis, replacement of the alveolar crest bone with fibrous tissue (flabby ridge), pyogenic granuloma, allergic reactions to prosthetic materials, and infectious reactions. As Junior specifies, many of the denture lesions see a rather recurrent risk factor, which is the hygienic maintenance of the prosthetic device $[35,36]$.

\section{DISCUSSION}

Despite the frequency of oral manifestations of gastrointestinal disease is variable and often nonspecific, oral lesions may still be a valuable aid in diagnosing gastrointestinal disorders and symptoms. The oral manifestations reported in GERD patients potentially involve both hard tissues and the oral cavity soft tissues. There is a clear evidence that these patients may suffer from dental erosion, soft tissue disorders, and decreased salivary flow volume [9]. Inspection of the oral cavity is always recommended to correlate this gastrointestinal disease with extra-esophageal signs and symptoms. Attention should be given to oral burning sensation and oral acidity, halitosis, erythema of hard and soft palate, and the uvula. As reported by Kainuma et al., the diagnosis of tongue mucosal disorders may serve as a screening for a presumptive diagnosis of GERD [47].

Dentistry should advise GERD patients to follow a proper, low-acid, and low-irritating diet, strict avoidance of eating just before going to bed, a cigarette smoking cessation program, a correct cervical-cephalic position during the sleep, a protection from the exposure to exogenous acidic factors in the workplace [48]. The more or less extensive enamel defects and soft tissue altered phenotype must also be prevented by eliminating all the suspected exacerbating factors that may easily become superimposed. Our therapy for GERD-related lingual manifestation made use of a diode laser. This approach has already presented excellent results in the management of mucosal lesions. The diode laser is particularly useful for small, prominent lesions such as the one we found on the tongue of our patient [49]. The hyperplasia of the filiform papillae in our patient may have determined, due to the increased thickness of the epithelium, a greater risk of suffering from halitosis. The tongue is primarily responsible for the phenomenon if it acts as a greater reservoir of microorganisms than other anatomical parts of the oral cavity. The action of the laser would presumably have reduced the lingual adverse effect in our patient suffering from GERD [50].

In this review, we decided to investigate, theoretically, the risk of self-induced oral injury in patients suffering from behavioral disorders of various origins. It is necessary to acquire knowledge about the most frequent organic causes that may lead to oral selfmutilation phenomena. The dentist must contribute to the multidisciplinary context of care of these patients.

Many of these patients are hospitalized, and a protective splint may already be prescribed in these circumstances. It is necessary to evaluate the mechanical properties of the intra-oral device because if poorly retained or stable on the insufficient residual dentition of the patients, it may inversely lead to a greater frequency of self-biting [25]. According to Okabe et al., it may be difficult for patients to keep wearing splints, and their application may create more stress. According to the authors, the difficulties of communication and the hospitalization environment in which the patients are treated can also affect their selfinjurious behaviors and paradoxically foster the phenomenon of self-biting [25].

The primary purpose of the above-mentioned therapeutic modality is the prevention of oral trauma to hard and soft tissues. Family members of patients with mental disorders should be informed about the importance of periodic visits to the dentist. According 
to Shim et al., there may be the necessity to sedate the patient before placing the intra-oral protective device [51]. In fact, while letting the patients wear it, the fingers of the dentists may be bitten. The patients may also break the devices. Parts of the broken devices may lead to respiratory problems. The bibliographic studies made by Arhakis et al., suggest the following devices as primary treatment options: lip-shields, lip bumbers, tongue protectors, occlusal spints and occlusal bite plates. All these can be modified to obtain the occlusal and the orthopedic modifications required for the clinical case in question [22].

As seen from this review, a common tissue reaction to poorly adapted dentures is inflammatory fibrous hyperplasia. As Mubarak reports, the lesion is mainly attributable to prosthetic factors or unbalanced occlusion [35]. In denture stomatitis, an erythema below the prosthetic base must be immediately identified to better settle the most appropriate therapy. The etiology is often multifactorial. Traumatic ulcers should always be correlated with prosthetic and nonprosthetic factors, to avoid a misdiagnosis of ulcerative lesion instead of oral carcinoma or any other oral pathology. According to Wahbi, patients provided with dentures that offer a valid and rewarding rehabilitation experience a better quality of life [5]. A careful analysis of prosthetic factors and local and systemic factors of patients should consistently prevent the risk of iatrogenic oral complications.

\section{CONCLUSION}

From this concise review and the clinical case, we have presented, it is clear that there is a clinical association between GERD and oral lesions. Oral complications and oral symptoms in GERD patients may include reduced masticatory function, reduced saliva flow volume, increased susceptibility to oral infection, dental erosion, and soft oral tissue disorders.

Patients with self-injurious behavior may inflict traumatic oral injury on themselves. An intra-oral protective device is one of the promising therapeutic options. Any intra-oral device installed in the mouth, along with a combination of prosthetic-iatrogenic, local and systemic factors, may result in oral complications. The removable dentures in denture wearers are also one example of such a case. An optimal fitting of the prosthesis and a good level of oral hygiene both in the mucosa and the prosthesis help reduce the risk of developing lesions in denture wearers.

As reported by Mejia M., referring to a concept already expressed in 2000 in the US Surgeon General's report Oral Health, the oral cavity is the mirror that reveals many of the inner secrets of our body [52].

\section{REFERENCES}

1. Rubenstein JH, Chen JW. Epidemiology of gastroesophageal reflux disease. Gastroenterology Clinics. 2014 Mar 1;43(1):1-4.

2. Anderloni A, Buri L, Cavallo G, Cestari R, Cipolletta L, Chilovi F, Costamagna G, Di Matteo G, Ficano L, Pietropaolo V, Ricci E. Impact of gastroesophageal reflux disease (GERD) on the Italian territor: The axis projet. Digestive and Liver Disease. 2006 Apr 1;38:S25-6.

3. Di Stasi F, Teruzzi C, Mantovani LG. PGS10 Cost of Illness Of Gastroesophageal Reflux Disease (GERD) in Italy. Value in Health. 2003 Nov 1;6(6):686-7.

4. Allareddy V, Lee MK, Da Silva JD, Rampa S, Allareddy V, Nalliah RP. Prevalence estimates and outcomes of mental health conditions in those hospitalized owing to dental conditions. Oral surgery, oral medicine, oral pathology and oral radiology. 2014 Sep 1;118(3):300-8.

5. Wahbi RH, Elamin EI. Impact of Removable Partial Denture on Quality-of-life of Sudanese Adults in Khartoum State. The journal of contemporary dental practice. 2018 Jan $1 ; 19(1): 102-8$.

6. Banerjee R, Chahande J, Banerjee S, Radke U. Evaluation of relationship between nutritional status and oral health related quality of life in complete denture wearers. Indian Journal of Dental Research. 2018 Sep 1;29(5):562-67.

7. Chatila AT, Nguyen MT, Krill T, Roark R, Bilal M, Reep G. Natural history, pathophysiology and evaluation of gastroesophageal reflux disease. Disease-a-Month. 2020 Jan 1;66(1):100848.

8. Yoshikawa H, Furuta K, Ueno M, Egawa M, Yoshino A, Kondo S, Nariai Y, Ishibashi H, Kinoshita Y, Sekine J. Oral symptoms including dental erosion in gastroesophageal reflux disease are associated with decreased salivary flow volume and swallowing function. Journal of gastroenterology. 2012 Apr 1;47(4):412-20.

9. Watanabe M, Nakatani E, Yoshikawa H, Kanno T, Nariai Y, Yoshino A, Vieth M, Kinoshita Y, Sekine J. Oral soft tissue disorders are associated with gastroesophageal reflux disease: retrospective study. BMC gastroenterology. 2017 Dec 1;17(1):92.

10. Vakil N, Van Zanten SV, Kahrilas P, Dent J, Jones R. The Montreal definition and classification of gastroesophageal reflux disease: a global, evidence-based consensus paper. Zeitschrift Fur Gastroenterologie. 2007 Nov 1;45(11):1125-40.

11. Lazarchik DA, Filler SJ. Dental erosion: predominant oral lesion in gastroesophageal reflux disease. The American journal of gastroenterology. 2000;95(8):S33-8.

12. Gregory-Head BL, Curtis DA, Kim L, Cello J. Evaluation of dental erosion in patients with 
gastroesophageal reflux disease. The Journal of prosthetic dentistry. 2000 Jun 1;83(6):675-80.

13. Bartlett DW, Evans DF, Anggiansah A, Smith BG. A study of the association between gastrooesophageal reflux and palatal dental erosion. British dental journal. 1996 Aug;181(4):125-31.

14. Picos A, Badea ME, Dumitrascu DL. Dental erosion in gastro-esophageal reflux disease. a systematic review. Clujul Medical. 2018 Oct;91(4):387-90.

15. Pace F, Pallotta S, Tonini M, Vakil N, Bianchi Porro G. Systematic review: gastro- oesophageal reflux disease and dental lesions. Alimentary pharmacology \& therapeutics. 2008 Jun;27(12):1179-86.

16. Li W, Liu J, Chen S, Wang Y, Zhang Z. Prevalence of dental erosion among people with gastroesophageal reflux disease in China. The Journal of prosthetic dentistry. 2017 Jan 1;117(1):48-54.

17. Tantbirojn D, Pintado MR, Versluis A, Dunn C, Delong R. Quantitative analysis of tooth surface loss associated with gastroesophageal reflux disease: a longitudinal clinical study. The Journal of the American Dental Association. 2012 Mar 1;143(3):278-85.

18. Song JY, Kim HH, Cho EJ, Kim TY. The relationship between gastroesophageal reflux disease and chronic periodontitis. Gut and liver. 2014 Jan;8(1):35-40.

19. Shimazu R, Yamamoto M, Minesaki A, Kuratomi Y. Dental and oropharyngeal lesions in rats with chronic acid reflux esophagitis. Auris Nasus Larynx. 2018 Jun 1;45(3):522-6.

20. Pirnat S. Versatility of an $810 \mathrm{~nm}$ diode laser in dentistry: an overview. J Laser Health Acad. 2007;4(2):1-9.

21. Limeres J, Feijoo JF, Baluja F, Seoane JM, Diniz M, Diz P. Oral self- injury. An update. Dental Traumatology. 2013 Feb;29(1):8-14.

22. Arhakis A, Topouzelis N, Kotsiomiti E, Kotsanos $\mathrm{N}$. Effective treatment of self- injurious oral trauma in Lesch-Nyhan syndrome: a case report. Dental Traumatology. 2010 Dec;26(6):496-500.

23. Cannavale R, Itro A, Campisi G, Compilato D, Colella G. Oral self-injuries: clinical findings in a series of 19 patients. Medicina Oral, Patologia Oral y Cirugia Bucal. 2015 Mar;20(2):e123-e129.

24. Saemundsson SR, Roberts MW. Oral selfinjurious behavior in the developmentally disabled: review and a case. ASDC journal of dentistry for children. 1997;64(3):205-209.

25. Okabe K, Kaneko R, Kawai T, Kano F, Ohta Y, Hibi H. Oral self-injury associated with septicaemia in a case of Cornelia de Lange syndrome. Journal of Oral and Maxillofacial Surgery, Medicine, and Pathology. 2020 Mar 1;32(2):136-9.

26. Siragusa M, Ferri R, Russo R, Lentini M, Schepis C. Self-inflicted lesions of the mouth and lips in mentally retarded young subjects. European Journal of Dermatology. 2013 Nov 1;23(6):843-8.

27. Kashyap RR, Kashyap RS. Self- inflicted injury as a potential trigger for carcinoma of lip-a case report. Gerodontology. 2013 Sep;30(3):236-8.

28. Riera-Punet N, Martinez-Gomis J, Zamora-Olave C, Willaert E, Peraire M. Satisfaction of patients with amyotrophic lateral sclerosis with an oral appliance for managing oral self-biting injuries and alterations in their masticatory system: A case-series study. The Journal of prosthetic dentistry. 2019 Apr 1;121(4):631-6.

29. Kondo K, Nakamura S, Kaneko T, Horie N, Shimoyama T. Unintentional self-injury of the tongue in an aged patient with Alzheimer's disease and behavioral and psychological symptoms of dementia, successfully treated with Kampo medicine: A case report. Journal of Oral and Maxillofacial Surgery, Medicine, and Pathology. 2016 Nov 1;28(6):551-4.

30. Kwon IJ, Kim SM, Park HK, Myoung H, Lee JH, Lee SK. Successful treatment of self-inflicted tongue trauma patient using a special oral appliance. International Journal of Pediatric Otorhinolaryngology. 2015 Nov 1;79(11):193841.

31. Santos MT, Manzano FS, Genovese WJ. Different approaches to dental management of self-inflicted oral trauma: Oral shield, botulinum toxin type A neuromuscular block, and oral surgery. Quintessence International. 2008 Feb 1;39(2):e639.

32. Shah RJ, Diwan FJ, Diwan MJ, Chauhan VJ, Agrawal HS, Patel GC. A study of the emotional effects of tooth loss in an edentulous Gujarati population and its association with depression. The Journal of the Indian Prosthodontic Society. 2015 Jul;15(3):237.

33. Jacobson TE, Krol AJ. A contemporary review of the factors involved in complete denture retention, stability, and support. Part I: retention. The Journal of prosthetic dentistry. 1983 Jan 1;49(1):515.

34. Budtz- Jørgensen E. Oral mucosal lesions associated with the wearing of removable dentures. Journal of Oral Pathology \& Medicine. 1981 Apr;10(2):65-80.

35. Mubarak S, Hmud A, Chandrasekharan S, Ali AA. Prevalence of denture-related oral lesions among patients attending College of Dentistry, University of Dammam: A clinico-pathological study. Journal of International Society of Preventive \& Community Dentistry. 2015 Nov;5(6):506.

36. Jefferson D, Melo de Matos DN, de Araujo J, John EL, de Vasconcelos. Analysis of oral injuries caused by the bad use of prosthesis -A literature review. International Journal of Development Research, 2017;7(8):14653-14657. 
37. Jainkittivong A, Aneksuk V, Langlais RP. Oral mucosal lesions in denture wearers. Gerodontology. 2010 Mar;27(1):26-32.

38. Jainkittivong A, Aneksuk V, Langlais RP. Oral mucosal conditions in elderly dental patients. Oral diseases. 2002 Jul;8(4):218-23.

39. Valente VB, Takamiya AS, Ferreira LL, Felipini RC, Biasoli ÉR, Miyahara GI, Bernabé DG. Oral squamous cell carcinoma misdiagnosed as a denture-related traumatic ulcer: a clinical report. The Journal of prosthetic dentistry. 2016 Mar 1;115(3):259-62.

40. Lee ST, Jang SB, Kwon TG, Choi SY. Oral tuberculosis mimicking a traumatic denture ulcer. The Journal of prosthetic dentistry. 2019 Feb $1 ; 121(2): 225-8$.

41. Zainal M, Zain NM, Amin IM, Ahmad VN. The antimicrobial and antibiofilm properties of allicin against Candida albicans and Staphylococcus aureus-A therapeutic potential for denture stomatitis. The Saudi Dental Journal. 2020 Feb 6.

42. Ramage G, Tomsett K, Wickes BL, López-Ribot JL, Redding SW. Denture stomatitis: a role for Candida biofilms. Oral Surgery, Oral Medicine, Oral Pathology, Oral Radiology, and Endodontology. 2004 Jul 1;98(1):53-9.

43. Abduljabbar T, Al-Askar M, Baig MK, AlSowygh $\mathrm{ZH}$, Kellesarian SV, Vohra F. Efficacy of photodynamic therapy in the inactivation of oral fungal colonization among cigarette smokers and non-smokers with denture stomatitis. Photodiagnosis and Photodynamic Therapy. 2017 Jun 1;18:50-3.

44. Badaró MM, Bueno FL, Arnez RM, de Cássia Oliveira V, Macedo AP, de Souza RF, Paranhos $\mathrm{HD}$, Silva-Lovato $\mathrm{CH}$. The effects of three disinfection protocols on Candida spp., denture stomatitis, and biofilm: A parallel group randomized controlled trial. The Journal of Prosthetic Dentistry. 2020 Jan 17; 124(6):690-98.

45. Ramage G, Tomsett K, Wickes BL, López-Ribot JL, Redding SW. Denture stomatitis: a role for Candida biofilms. Oral Surgery, Oral Medicine, Oral Pathology, Oral Radiology, and Endodontology. 2004 Jul 1;98(1):53-9.

46. Alrabiah M, Alsahhaf A, Alofi RS, Al-Aali KA, Abduljabbar T, Vohra F. Efficacy of photodynamic therapy versus local nystatin in the treatment of denture stomatitis: A randomized clinical study. Photodiagnosis and Photodynamic Therapy. 2019 Dec 1;28:98-101.

47. Kainuma M, Furusyo N, Urita Y, Nagata M, Ihara T, Nakaguchi T, Namiki T. Mo1130 The Association Between Objective Tongue Color and Gastroesophageal Reflux Disease. Results From the Kyushu and Okinawa Population Study (KOPS). Gastroenterology. 2015 Apr 1;148(4):S615.

48. Dahshan A, Patel H, Delaney J, Wuerth A, Thomas R, Tolia V. Gastroesophageal reflux disease and dental erosion in children. The Journal of pediatrics. 2002 Apr 1;140(4):474-8.

49. Azma E, Safavi N. Diode laser application in soft tissue oral surgery. Journal of lasers in medical sciences. 2013;4(4):206-11.

50. Krespi YP, Kizhner V, Wilson KA, Sivriver A, Low S, Khosravi Y, Stoodley P. Laser tongue debridement for oral malodor-A novel approach to halitosis. American Journal of Otolaryngology. 2020 Mar 13:102458.

51. Shim SH, Ahn GS. Treatment of Self-Injurious Oral Trauma in Patient with Cerebral Palsy: A Case Report. International Journal of Clinical Preventive Dentistry. 2017 Sep 30;13(3):155-8.

52. Mejia LM. Oral manifestations of gastrointestinal disorders. Atlas Oral Maxillofac Surg Clin North Am. 2017 Sep 1;25(2):93-104. 